\title{
Metabolic Lysis of Yeast Protoplasts
}

\author{
By K. J. INDGE \\ Department of Biochemistry, University of Manchester Institute of \\ Science and Technology, Manchester
}

(Accepted for publication 26 October 1967)

\begin{abstract}
SUMMAR Y
Yeast protoplasts which were in osmotic equilibrium with a $0.55 \mathrm{M}$-mannitol solution underwent lysis when glucose was added to the suspension. Lysis was dependent on glucose metabolism and was accelerated when chelating agents were added to the medium. Lysis in the presence of glucose and chelating agents was inhibitied by alkali metal ions, magnesium, spermidine and by increasing the tonicity of the test medium. The inhibition of lysis by $\mathrm{K}^{+}$and $\mathrm{Na}^{+}$when chelating agents were present conformed to a MichaelisMenten type relationship with apparent dissociation constants of $2-5 \times 10^{-4}$ $\mathrm{M}$ and $3^{-6} \times \mathrm{IO}^{-3} \mathrm{M}$ for $\mathrm{K}^{+}$and $\mathrm{Na}^{+}$, respectively.
\end{abstract}

\section{INTRODUCTION}

While the growth and metabolism of bacterial and yeast protoplasts has been examined by various workers (McQuillen, I960; Jeynes, I96I; Shockman \& Lampen, 1962; Eddy \& Williamson, 1959; Svoboda \& Necas, 1966; Gascon, Ochoa \& Villanueva, 1965), less attention has been paid to the circumstances in which the protoplasts may lyse as a result of their own metabolic activities (Abrams, 1959, 1960; Wachsman \& Storck, 1960). In the present paper the influence of the composition of the test medium on the osmotic properties of metabolizing yeast protoplasts has been examined. The results show that in the presence of glucose the protoplasts tend to undergo metabolic lysis (Abrams, 1959) and that in short-term experiments the extent of lysis of the population is largely determined by the ionic composition of the test medium.

\section{METHODS}

Organism. Stock suspensions of protoplasts of the yeast Saccharomyces carlsbergensis NCYC 74 were prepared as described in the previous paper (Indge, 1968).

Experimental techniques. Samples $\left(0.05^{-0} .10 \mathrm{ml}\right.$.) of the stock suspension of protoplasts were added to $5 \mathrm{ml}$. of a basal test medium containing mannitol (10\%, w/v) and $0.0 \mathrm{I}$ M-imidazole $\mathrm{HCl}$ buffer $(\mathrm{pH} \mathrm{6.4})$. The test solutions were equilibrated at room temperature $\left(25^{\circ}\right)$ during ro min. and $0.25 \mathrm{ml}$. of glucose solution (IO $\%, \mathrm{w} / \mathrm{v}$ ) were then added to start the experiment. Extinction changes with time in the test samples were followed in a Hilger Spekker using neutral filters (Ilford, H. 508) and I cm. cells.

Compounds which were added to the basal test medium were made up in $10 \%$ (w/v) mannitol solution containing $0.0 \mathrm{I}$ M-imidazole $\mathrm{HCl}$ buffer ( $\mathrm{pH} \mathrm{6.4)}$ ). Cations were added as the chlorides and anions as the tris salts. 
Carbon dioxide evolution and oxygen consumption were measured by standard manometric techniques. When the respective rates of glucose metabolism and lysis were to be correlated the measurements were done in parallel. To estimate the initial rates of glucose metabolism in tests in which rapid rates of lysis were obtained, the suspension density of the protoplasts in the manometer flasks was increased threefold. $\mathrm{Q}$ values are reported in terms of the equivalent dry weight of whole yeast.

\section{RESULTS}

\section{The influence of glucose}

It can be seen from Fig. I that the extinction values of protoplast suspensions decreased only slowly with time during incubations in the absence of glucose. The addition of glucose $(0.5 \%, \mathrm{w} / \mathrm{v})$ produced marked decreases in the extinctions of the suspensions, but when $\mathrm{KCl}(\mathrm{O} \cdot \mathrm{O} \mathrm{I})$ was also included in the medium the extinctions subsequently increased. This latter change was accompanied by aberrant cell-wall synthesis, swelling and growth of the protoplasts as described by Eddy \& Williamson (I959). Sodium chloride (O.0I M) as opposed to $\mathrm{KCl}$ was found to decrease only slightly the extinction changes induced by glucose. When glucose alone was added all the protoplasts were lysed.

\section{The effects of chelating agents}

When glucose was added to osmotically stabilized protoplast suspensions containing citrate, ATP, EDTA or pyrophosphate (each $0.0 \mathrm{I}$ M) a rapid decrease in the extinctions of the mixtures ensued. This finding is illustrated by the results of an experiment in which citrate was added as the chelating agent (Fig. 2). Experience showed that a rapid decrease in the extinctions of the protoplast suspensions resulted only when both glucose and a chelating agent were added (Fig. 2). When the protoplasts were incubated with glucose for up to $30 \mathrm{~min}$. before the addition of citrate, results similar to those of Fig. 2 were obtained.

Previous observations (Indge, 1968) had shown that in protoplast suspensions subjected to osmotic shock there was a linear relationship between the extinction change and the number of protoplasts lysed. A similar correlation between extinction changes and lysis of the protoplasts was found in the present experiments. When a suspension of metabolizing protoplasts was cooled to $0^{\circ}$ (see later) and the number of intact protoplasts counted by using a Neubauer counting chamber, a linear relationship was found between the number of protoplasts lysed and the observed extinction change for a range of experimental conditions. Thus the extinction changes recorded in these experiments are a measure of protoplast lysis.

The relation between the rate of extinction decrease after the addition of glucose to the protoplast suspensions and the concentration of citrate, EDTA, ATP or pyrophosphate in the medium was investigated in a series of ten experiments. Representative data are given in Table I. Analyses of the results of these experiments showed that in no instance did the kinetics conform to a Michaelis-Menten type relationship.

\section{The role of glucose}

The rate of lysis of protoplasts which were suspended in $10 \%(\mathrm{w} / \mathrm{v})$ mannitol solution + citrate was influenced by the amount of glucose added. An analysis of the effects of glucose concentration on lysis showed that the initial rate of lysis of proto- 
plast populations was proportional to the initial rate of glucose metabolism (Table 2). Parallel experiments done under nitrogen, or with Antimycin A ( $15 \mu \mathrm{g} . / \mathrm{ml}$.) added to the medium to suppress oxidative metabolism of the protoplasts, showed that while glucose respiration appeared to contribute significantly to the net rate of lysis, lysis did not depend absolutely on respiration. A point of interest emerging from these results

Table I. The stimulation of metabolic lysis of yeast protoplasts by chelating agents

Protoplasts were suspended in $0.01 \mathrm{M}$-imidazole $\mathrm{HCl}$ buffer (pH 6.4) containing mannitol $(10 \%, \mathrm{w} / \mathrm{v})$, glucose $(0.5, \mathrm{w} / \mathrm{v})$ and certain chelating anions. The rate of protoplast lysis is expressed as the extinction decrease $/ 5 \mathrm{~min}$. observed after the addition of glucose.

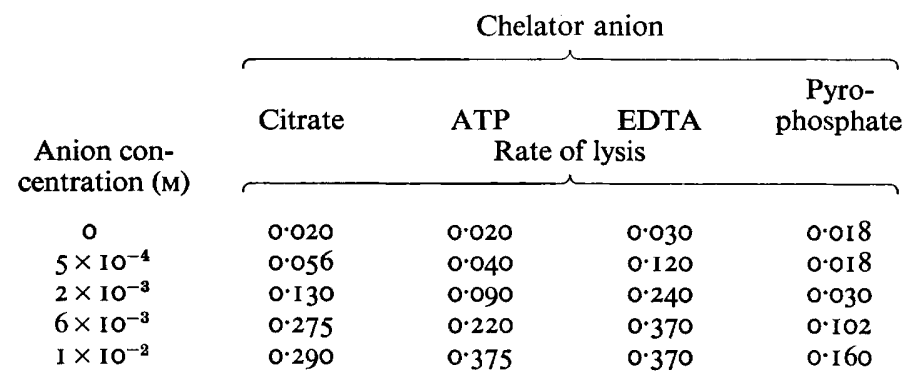

Table 2. The relationship between glucose metabolism and yeast protoplast lysis

The suspension medium contained $0.0 \mathrm{I}$-imidazole $\mathrm{HCl}$ buffer (pH 6.4), mannitol $(10 \%, \mathrm{w} / \mathrm{v})$ and citrate $(6 \mathrm{~mm})$. The results are from parallel experiments in which extinction changes and gas exchanges were measured as a function of glucose concentration either with $(+)$ or without $(-)$ Antimycin A. Lysis rates expressed as extinction decrease/5 min. and $\mathrm{Q}$ values as $\mu \mathrm{l} . / \mathrm{hr}$./mg. dry wt yeast.

$\begin{array}{lcc}\begin{array}{c}\text { Glucose added } \\ (\%, \mathrm{w} / \mathrm{v})\end{array} & \text { Lysis rate } & Q_{\mathrm{Ci}}^{\mathrm{Air}} \\ 0 & 0.0 \mathrm{I} & 0 \\ 0.005 & 0.054 & 25 \\ 0.01 & 0.083 & 43 \\ 0.025 & 0.139 & 50 \\ 0.10 & 0.270 & 68 \\ \mathrm{I} \cdot 0 & 0.270 & 70 \\ 0 & 0.001 & 0 \\ 0.005 & 0.022 & 40 \\ 0.0 \mathrm{I} & 0.050 & 75 \\ 0.025 & 0.068 & 90 \\ 0.10 & 0.115 & \mathrm{I} 25 \\ \mathrm{I} \cdot 0 & 0.120 & 128\end{array}$

Antimycin A
(I5 $\mu \mathrm{g} . / \mathrm{ml}$.
-
-
-
-
-
-
+
+
+
+
+
+

was that Antimycin A or anaerobiosis inhibited the slow extinction change usually observed in endogenous tests (see Figs. I, 2). Several carbohydrates other than glucose were tested for ability to promote lysis in the presence of chelating agents. Of sixteen compounds tested only those which were metabolized by the protoplasts, namely sucrose, fructose and mannose, induced lysis when added to the medium at a concentration of $\mathrm{I} \%(\mathrm{w} / \mathrm{v})$. 


\section{Inhibition of metabolism}

Concentrations of iodoacetate or iodoacetamide (0.005-0.0I M) which were sufficient to inhibit glucose metabolism completely also inhibited lysis of the protoplasts in the presence of chelating agents + glucose. The detailed effects of these inhibitors were however complex. For instance, when glucose and iodoacetamide $\left(\mathrm{I}-5 \times \mathrm{IO}^{-3} \mathrm{M}\right)$ were added to the protoplasts together, the rate of lysis was increased twofold. Incubation of the protoplasts with iodoacetamide $\left(\mathrm{I}-5 \times \mathrm{IO}^{-3} \mathrm{M}\right)$ during $20 \mathrm{~min}$. before the addition of glucose resulted in either stimulation or inhibition of lysis, depending on the concentration of inhibitor. Sodium azide $\left(5 \times \mathrm{IO}^{-4} \mathrm{M}\right)$ and 2,4-dinitrophenol $\left(2 \times 10^{-3} \mathrm{M}\right)$ completely inhibited the lysis reaction induced by the addition of glucose and citrate or EDTA to the protoplast suspensions.

The initial rates of lysis (extinction decrease per $5 \mathrm{~min}$.) at $0^{\circ}, 10^{\circ}, 20^{\circ}$ and $28^{\circ}$ were $0.00,0.03,0.12$ and 0.30 , respectively, in one test done with glucose $(0.5 \% \mathrm{w} / \mathrm{v})+$ citrate $(6 \mathrm{mM})$.

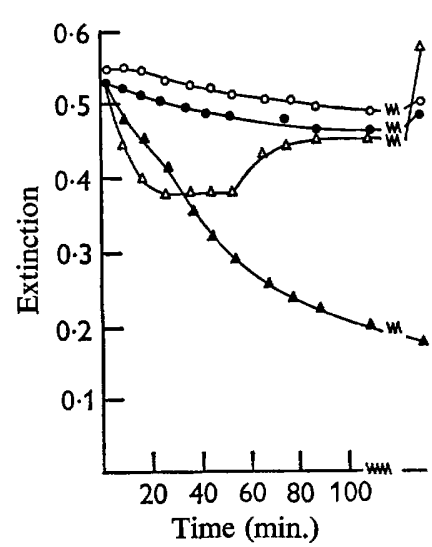

Fig. I

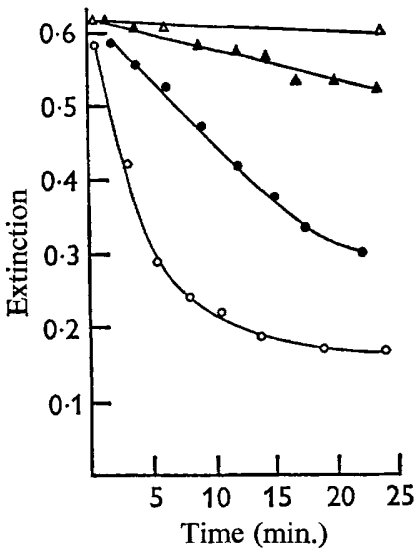

Fig. 2

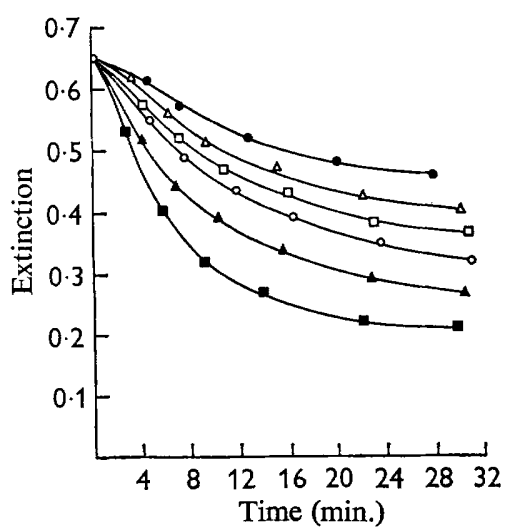

Fig. 3

Fig. I. The effects of glucose and $\mathrm{K}^{+}$on yeast protoplasts. Protoplasts were incubated in $0.01 \mathrm{M}$-imidazole $\mathrm{HCl}$ buffer ( $\mathrm{pH} \mathrm{6.4)}$, mannitol $(10 \%$, w/v) and glucose $(0.5 \%$, w/v) either with $(\triangle)$ or without $(\triangle) \mathrm{KCl}(0.0 \mathrm{I} \mathrm{M})$. Tests with $(\bullet)$ and without $(\mathrm{O}) \mathrm{KCl}(0.0 \mathrm{M} \mathrm{M})$ in the absence of glucose. The broken lines signify further incubation for $16 \mathrm{~h}$.

Fig. 2. Lysis of yeast protoplasts by the addition of glucose and citrate ions. Tests made in $0.01 \mathrm{M}$-imidazole $\mathrm{HCl}$ buffer $(\mathrm{pH} 6.4)$ with $10 \%(\mathrm{w} / \mathrm{v})$ mannitol + glucose $(0.5 \%$, w/v) added. Citrate concentration $0.006 \mathrm{M}(\mathrm{O})$; $0.00 \mathrm{I} \mathrm{M}(\mathbf{\bullet})$; zero $(\boldsymbol{\Delta})$. Control test with citrate $(0.006 \mathrm{M})$ but no glucose $(\triangle)$.

Fig. 3. Inhibition of lysis of yeast protoplasts by monovalent cations. The medium contained $0.01 \mathrm{M}$-imidazole $\mathrm{HCl}$ buffer $(\mathrm{pH} 6.4)$, mannitol $(10 \%, \mathrm{w} / \mathrm{v})$, glucose $(0.5 \%, \mathrm{w} / \mathrm{v})$, citrate $(0.004 \mathrm{M})$ and the chloride of the cation under test $(0.0 \mathrm{I} \mathrm{M}) . \mathrm{NH}_{4}+\mathbf{0} ; \mathrm{K}^{+} \triangle ; \mathrm{Rb}^{+} \square$; $\mathrm{Cs}^{+} \mathrm{O} ; \mathrm{Na}^{+}$and $\mathrm{Li}^{+} \mathbf{\Delta}$ (these cations yielded coincident curves); control test $\mathbf{a}$

\section{Effects of cations}

As can be seen from Fig. 3 monovalent cations (O.OI M) exhibited graded abilities to inhibit the rapid lysis of the protoplasts induced by citrate and glucose, $\mathrm{NH}_{4}{ }^{+}$ and $\mathrm{K}^{+}$being the most effective inhibitors. Of the cations tested $\mathrm{Na}^{+}$and $\mathrm{K}^{+}$were selected for more detailed studies. The relation between the rate of lysis and the concentration of $\mathrm{K}^{+}$or $\mathrm{Na}^{+}$in the medium was investigated. The results from a typical 
experiment are shown in Fig. 4. It can be readily shown that when a cation binding site which controls the rate of lysis is inactivated by the formation of a reversible complex with monovalent cations, then the decrease in the rate of lysis in the presence of cations and the cation concentration would be related by a Michaelis-Menten type equation. When the kinetic data were plotted according to the procedure of Hofstee (1952) they yielded straight lines (Fig. 5). Thus the results conformed to the type of relationship suggested.

The apparent dissociation constants of the presumed $\mathrm{Na}^{+}$and $\mathrm{K}^{+}$complexes determined from the results of twelve experiments varied between $3-6 \times 10^{-3} \mathrm{M}$ and $2-5 \times$ $\mathrm{IO}^{-4} \mathrm{M}$, respectively. These values were apparently independent of the nature or concentration of the chelating agent employed to stimulate the lysis reaction (Fig. 5). The results presented in Fig. 5 were obtained from measurements of the rates of extinction change in short-term experiments where the average rate of lysis was large (see Fig. 4). When the rate of lysis was small, for example in tests carried out with $0-2$ mm-citrate, the addition of $\mathrm{K}^{+}$to the medium tended to increase the rate of lysis (see Fig. I).

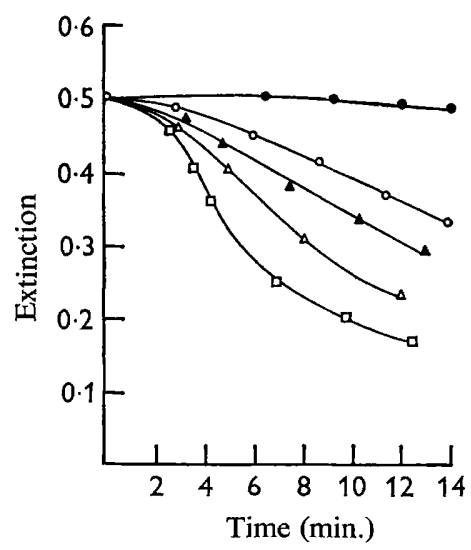

Fig. 4

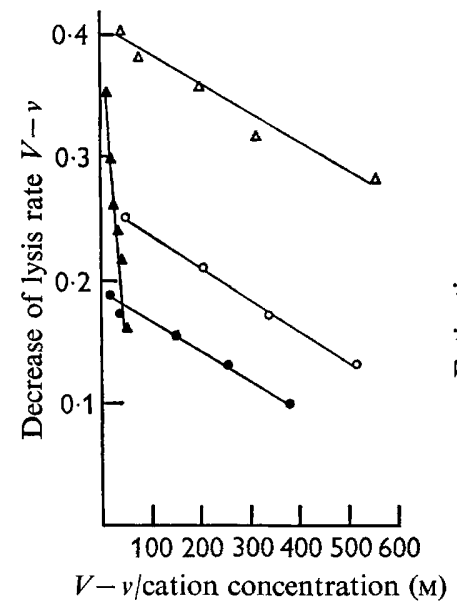

Fig. 5

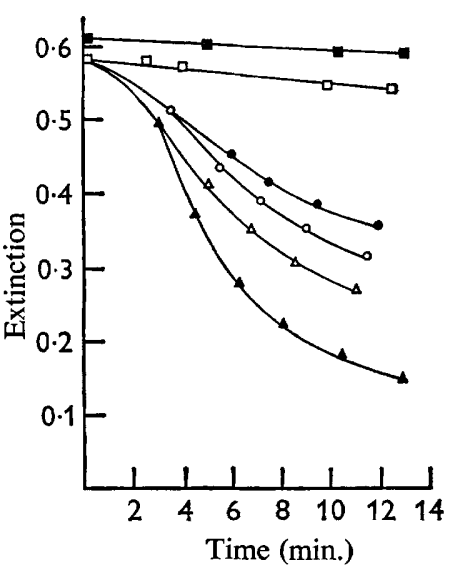

Fig. 6

Fig. 4. Kinetics of inhibition of yeast protoplast lysis by $\mathrm{K}^{+}$. Test conditions as for Fig. 3 . $\mathrm{KCl} 0.00 \mathrm{I} \mathrm{M,} \mathrm{O;0.0005} \mathrm{M,} \mathbf{\Delta} ; 0.00025 \mathrm{M}, \triangle$; zero, $\square$. Control test with no citrate or $\mathrm{K}^{+}$, $\bullet$.

Fig. 5. Inhibition of yeast protoplast lysis by $\mathrm{Na}^{+}$and $\mathrm{K}^{+}$. The rate of lysis $(V)$ in tests without $\mathrm{Na}^{+}$or $\mathrm{K}^{+}$minus the rate of lysis $(v)$ in tests with $\mathrm{Na}^{+}$or $\mathrm{K}^{+}$is plotted against $V-v /$ cation concentration. Lysis rates were estimated as the extinction decrease $/ 5 \mathrm{~min}$. The rates of lysis observed with no $\mathrm{K}^{+}$or $\mathrm{Na}^{+}$added are given below; note that in all cases the plots for $\mathrm{K}^{+}$cut the ordinate axis at values less than $V$ (see text). $0.004 \mathrm{M}$-EDTA and $\mathrm{K}^{+}, \triangle$ $(V=0.50)$; $0.006 \mathrm{M}$-citrate and $\mathrm{K}^{+}, \mathrm{O}(V=0.44)$; $0.004 \mathrm{M}$-citrate and $\mathrm{K}^{+}, \mathrm{O}(V=0.25)$; $0.006 \mathrm{M}$-citrate and $\mathrm{Na}^{+}, \boldsymbol{\Delta}(V=0.40)$.

Fig. 6. The effect of the concentration of mannitol in the medium on lysis of yeast protoplasts. The media contained $0.01 \mathrm{M}$-imidazole $\mathrm{HCl}$ buffer $(\mathrm{pH} 6.4)$, glucose $(0.5 \%, \mathrm{w} / \mathrm{v})$, citrate (O.OI M) and I4 (O), I3 $(0), I_{2}(\triangle)$ and Io $(\Delta) \%(w / v)$ mannitol. Control tests without citrate added in $\mathrm{I} 4(\mathbf{G})$ and $\mathrm{IO}(\square) \%(\mathrm{w} / \mathrm{v})$ mannitol.

$\mathrm{Na}^{+}$did not stimulate lysis appreciably under such conditions. Another aspect of the complex behaviour exhibited towards $\mathrm{K}^{+}$was that it did not annul completely the rapid lysis reaction induced by chelating agents and glucose (Fig. 4). Comparison of the intercepts on the ordinate axis of the plots of Fig. 5 with the rates of lysis of the 
respective control tests also demonstrates this point. A possible explanation is that the protoplasts transport and accumulate $\mathrm{K}^{+}$during the experiments thereby increasing their osmotic pressure relative to that of the medium and thus increasing the rate of lysis.

Spermidine ( $\left(\mathrm{IO}^{-5}\right.$ to $\left.\mathrm{IO}^{-4} \mathrm{M}\right)$ and $\mathrm{Mg}^{2+}\left(\mathrm{IO}^{-4}\right.$ to $\left.\mathrm{IO}^{-3} \mathrm{M}\right)$ decreased the rate of lysis of the protoplasts in the presence of citrate $(6 \mathrm{~mm})$ and glucose by up to $50 \%$. Their effects were not investigated in detail, but even when excess citrate $(6 \mathrm{mM})$ was present $\mathrm{Mg}^{2+}\left(\mathrm{IO}^{-4} \mathrm{M}\right)$ decreased the rate of lysis by about $40 \%$.

\section{Osmotic pressure of the medium}

In the osmotic lysis tests described in a previous report (Indge, 1968) chelating agents were shown to lower the resistance of yeast protoplasts to lysis by osmotic shock by an amount equivalent to a $2 \cdot 5 \%$ (w/v) decrease in the concentration of mannitol in the suspending fluid. As shown in Fig. 6 the stimulation of the lysis of metabolizing protoplasts observed when citrate was included in the medium was not prevented even in $14 \%(\mathrm{w} / \mathrm{v})$ mannitol solution. Increasing the concentration of mannitol in the medium to $14 \%(\mathrm{w} / \mathrm{v})$ did not decrease the rate at which the protoplasts metabolized glucose.

\section{The rate of lysis}

Although the effects of chelating agents described were qualitatively highly reproducible, the rate of lysis varied somewhat from one batch of protoplasts to another. For example, the initial rates of lysis recorded in citrate solution (6 mM) and glucose varied from 0.15 to 0.45 (extinction decrease $/ 5 \mathrm{~min}$.), the average value being about 0.30 , in a set of 30 measurements. A lag period of 5-10 min. after the addition of glucose was sometimes observed before lysis commenced.

\section{Table 3. The influence of anions on yeast protoplast lysis}

Tests were made in media containing 0.01 M-imidazole $\mathrm{HCl}$ buffer ( $\mathrm{pH} \mathrm{6.4}$ ), mannitol $(10 \%, \mathrm{w} / \mathrm{v})$ and glucose $(0.5 \%, \mathrm{w} / \mathrm{v})$. Lysis rates, which are expressed as the extinction decrease $/ 5$ min., were measured over a period of linear extinction change with time after the lag periods (see text).

$\begin{array}{cccc}\begin{array}{c}\text { Anion con- } \\ \text { Antration (mM) }\end{array} & \begin{array}{c}\text { Succinate } \\ \text { lysis rate }\end{array} & \text { Acetate } \\ 0 & 0.036 & 0.046 & 0.040 \\ 2 & 0.056 & 0.086 & 0.065 \\ 4 & 0.068 & 0.110 & 0.100 \\ 6 & 0.097 & 0.184 & . \\ 8 & 0.136 & 0.184 & 0.125 \\ 10 & 0.136 & 0.184 & 0.140\end{array}$

Preparations of protoplasts which lysed rapidly in the presence of glucose and strong chelating agents were also susceptible to lysis when weaker complexing agents such as sulphate, phosphate, acetate, succinate, malate or aconitate were added. The concentrations of these anions resulting in rapid rates of lysis were in each case between $10^{-3} \mathrm{M}$ and $1 \mathrm{O}^{-2} \mathrm{M}$ (Table 3 ). Their effects were characterized by lag periods of up to $20 \mathrm{~min}$. before any appreciable stimulation of lysis took place. In some instances it 
was necessary to decrease the concentration of mannitol to $8.5 \%(\mathrm{w} / \mathrm{v})$ in order to observe the effects, of these anions on lysis conveniently. The actions of these anions on lysis were inhibited by $\mathrm{K}^{+}, \mathrm{Na}^{+}$and $\mathrm{Mg}^{2+}$.

\section{DISCUSSION}

The lysis of yeast protoplasts induced by glucose resembles the phenomenon of metabolic lysis observed by Abrams (1959, 1960) with Streptococcus faecalis protoplasts, in that the reaction depends on glucose metabolism. Certain properties of the yeast system, however, differ from those of the bacterial system studied by Abrams. In the yeast protoplast experiments reversible metabolic swelling did not appear to take place, and monovalent cations inhibited rather than stimulated lysis. The evidence indicated that $\mathrm{K}^{+}$tended to promote lysis of the yeast protoplasts, but this action was generally masked by the net inhibition of lysis produced by this cation.

The present results support the view proposed by Abrams that the addition of an energy source to protoplasts causes a change in the permeability properties of the protoplast membrane so that the osmotic stabilizer enters the cells. In yeast protoplasts this change appears to be accentuated by chelating agents, so that the rate of metabolic lysis is greatly increased. Lehninger (1962) discussed the similarities which exist between the swelling and contraction phenomena observed in mitochondria and in the protoplasts of micro-organisms.

It is of interest to compare the present results with those of the previous osmotic lysis experiments (Indge, I968). Metabolizing yeast protoplasts were susceptible to the action of citrate ion up to $30 \mathrm{~min}$. after the addition of glucose, which contrasts with the transient susceptibility of resting protoplasts to chelating agents during osmotic shock tests. Anions such as phosphate and succinate, which bind $\mathrm{Mg}^{2+}$ less strongly than do citrate or EDTA (Sillén \& Martell, 1964), increased the rate of lysis in metabolic tests but did not influence the osmotic lysis of the protoplasts. These observations, and also the apparently larger effects of citrate ion on the stability of the protoplasts in the metabolic as opposed to the osmotic lysis tests (Fig. 6), can be explained in terms of the hypothesis put forward in the preceding paper (Indge, 1968). There it was suggested that bound $\mathrm{Mg}^{2+}$ or other cations in some way controlled the permeability properties of the protoplast membrane. Therefore it is proposed that in metabolizing protoplasts the $\mathrm{Mg}^{2+}$ of the membrane is accessible to the medium, the conformation and properties of the membrane being those which are exhibited during osmotic swelling of the protoplasts.

A further difference between the properties of the protoplasts in the two test systems is that the affinity of the membrane cation-binding site postulated to account for the inhibition of metabolic and osmotic lysis by $\mathrm{Na}^{+}$and $\mathrm{K}^{+}$was increased when the protoplasts were metabolizing glucose. The similarity between the apparent dissociation constants of the presumed $\mathrm{Na}^{+}$and $\mathrm{K}^{+}$complexes in metabolically active protoplasts and those of the cation transport system in yeast (Armstrong \& Rothstein, I964, I967) leads one to suspect that these phenomena may be related. One interesting possibility is that the non-transporting modifier site postulated by Armstrong \& Rothstein (1967) is involved in the permeability changes described in the present investigation. The phenomenon of metabolic lysis may be of practical importance in preparing yeast protoplasts. The results of the present work indicate that buffer 
solutions containing chelating agents such as citrate should be avoided, and that the yeasts which exhibit high values of endogenous respiration incubations with snail-gut juice should be done anaerobically.

The author is indebted to Professor A. A. Eddy for valuable critical discussions and to $\mathrm{Mr} \mathrm{N}$. B. Roberts for his technical assistance.

\section{REFERENCES}

Abrams, A. (1959). Reversible metabolic swelling of bacterial protoplasts. J. biol. Chem. 234, 383 .

ABRAMS, A. (1960). Metabolically dependent penetration of oligosaccharides into bacterial cells and protoplasts. J. biol Chem. 235, I28I.

Armstrong, W. McD. \& Rothstein, A. (1964). Discrimination between alkali metal cations by yeast. I. Effect of $\mathrm{pH}$ on uptake. J. gen. Physiol. 48, 6I.

Armstrong, W. MCD. \& Rothstein, A. (I967). Discrimination between alkali metal cations by yeast. II. Cation interactions in transport. J. gen. Physiol. 5o, 967.

EDDY, A. A. \& Williamson, D. H. (1959). Formation of aberrant cell walls and of spores by the growing yeast protoplast. Nature, Lond. I83, IIOI.

Gascon, A., Ochoa, A. G. \& Villanueva, J. R. (I965). Production of yeast and mould protoplasts by treatment with the strepzyme of Micromonospora AS. Canad. J. Microbiol. II, 573.

HofsteE, B. H. J. (1952). On the evaluation of the constants $V_{m}$ and $K_{m}$ in enzyme reactions. Science, N.Y. II6, 3029.

INDGE, K. J. (I968). The effects of various anions and cations on the lysis of yeast protoplasts by osmotic shock. J. gen. Microbiol. 5I, 425.

JEYNES, M. H. (196I). The growth and division of bacterial protoplasts. Expl Cell Res. 24, 255.

LEHNINGER, A. L. (1962). Water uptake and extrusion by mitochondria in relation to oxidative phosphorylation. Physiol. Rev. 42, 467.

McQuillen, K. (1960). In The Bacteria. Ed. by I. C. Gunsalus and R. Y. Stanier, Vol. I, p. 249. New York and London: Academic Press.

SHockman, G. D. \& LAMPEN, J. O. (1962). Inhibition by antibiotics of the growth of bacterial and yeast protoplasts. J. Bact. 84, 508.

Sillén, L. G. \& MARTell, A. E. (1964). Stability constants of metal-ion complexes. Special Publication, no. 17. London: The Chemical Society.

SvobodA, A. \& NeČAS, O. (I966). Regeneration of yeast protoplasts prepared by snail enzyme. Nature, Lond. 210, 845 .

WACHSMAN, J. T. \& Storck, R. (1960). Propionate induced lysis of protoplasts of Bacillus megaterium. J. Bact. 8o, 600 . 